\title{
Increased mucosal CD4+ T-cell activation following vaccination with an adenoviral vector in rhesus macaques
}

\author{
I Bukh*, R Calcedo, S Roy, DG Carnathan, R Grant, SJ Ratcliffe, JM Wilson, MR Betts \\ From AIDS Vaccine 2012 \\ Boston, MA, USA. 9-12 September 2012
}

\section{Background}

The possibility that vaccination with Adenoviral vectors increased mucosal $\mathrm{T}$-cell activation remains a central hypothesis to explain the potential enhancement of HIV acquisition within the STEP trial. Modeling this within rhesus macaques is complicated because human Adenoviruses, including Adenovirus type 5 (HAd5), do not productively infect macaques. We created a vector based upon a naturally occurring rhesus macaque Adenovirus (SAdV7) to test whether vaccination with a species-specific Adenoviral vector enhances mucosal T-cell activation within the natural host.

\section{Methods}

Twelve rhesus macaques were vaccinated $3 \mathrm{x}$ intramuscularly with SAdV7 vector. Five HAd5-vaccinated animals were included as controls. PBMC and rectal biopsies were obtained at baseline, multiple times post-prime and post17 week boost ( $8 \mathrm{x} / \mathrm{animal})$, and post-31 week second boost (1x/animal). We assessed rectal mucosal lamina propria and blood for frequency changes of Ad-specific T-cell responses and $\mathrm{T}$-cell activation levels by measuring IFNg, TNFa, IL2, CD25, Ki67, CD69, and HLA-DR.

\section{Results}

Naturally acquired pre-existing SAdV7-specific CD4+ Tcells were identified in 10/13 macaques within blood and/or rectal mucosa. Following intramuscular SAdV7 vaccination, rectal SAdV7-specific CD4+ T-cell responses increased above baseline in 9/9 animals $2-5$ weeks postprime, and subsequently contracted. Five weeks postprime, 10/12 animals had rectal SAdV7-specific CD4+ $\mathrm{T}$-cell responses ranging from $0.1-16.84 \%$. As expected,
SAdV7-specific CD4+ T-cells expressed CD69 and other activation markers (but not Ki67). Heightened expression of CD25, CD69, and HLA-DR was observed on total rectal memory CD4+ T-cells in SAdV7-vaccinated animals, and maintained 15 weeks after the prime. Interestingly, upregulation of activation markers in rectal mucosa also occurred in HAd5-vaccinated animals. No change in activation was observed in the blood throughout the entire study.

\section{Conclusion}

These results indicate that peripheral vaccination with an Adenovirus vector can increase the activation of mucosal CD4+ T-cells providing an experimental model to further evaluate the role of host-vector interactions on increased HIV acquisition.

Published: 13 September 2012

doi:10.1186/1742-4690-9-S2-P267

Cite this article as: Bukh et al:: Increased mucosal CD4+ T-cell activation following vaccination with an adenoviral vector in rhesus macaques. Retrovirology 2012 9(Suppl 2):P267.

University of Pennsylvania, Philadelphia, PA, USA

C 2012 Bukh et al; licensee BioMed Central Ltd. This is an Open Access article distributed under the terms of the Creative Commons 